\title{
Simulating hydrogen in fcc materials with discrete dislocation plasticity
}

\author{
Haiyang $\mathrm{Yu}^{\mathrm{a}, *}$, Alan Cocks ${ }^{\mathrm{b}}$, Edmund Tarleton ${ }^{\mathrm{b}, \mathrm{a}}$ \\ ${ }^{a}$ Department of Materials, University of Oxford, Parks Road, OX1 3PH, UK \\ ${ }^{b}$ Department of Engineering Science, University of Oxford, Parks Road, OXI 3PJ, UK
}

\begin{abstract}
We have performed discrete dislocation plasticity simulations of hydrogen charged microcantilever bend tests on an fcc material at realistic hydrogen concentrations. This was achieved by accounting for the near-core solute-solute interactions which was found to reduce the dislocation nucleation time and stress. Dislocation pile-ups were observed at the neutral mid plane of the cantilever, and hydrogen was found to increase the number of dislocations in the pile-ups. Meanwhile, hydrogen was observed to decrease the flow stress due to the reduced dislocation core force. This was in contrast to the first-order hydrogen elastic shielding mechanism which was found to be negligible at realistic concentrations. Local stress elevation was observed in the presence of hydrogen in simulations which included an obstacle close to the free surface of the microcantilever, indicating how hydrogen might induce premature stress controlled failure.
\end{abstract}

Keywords: Hydrogen embrittlement, discrete dislocation dynamics, hydrogen enhanced localized plasticity, fcc material

DOI: $10.1016 / j$. ijhydene.2020.01.118

\section{Introduction}

Hydrogen embrittlement (HE), a reduction in material strength and ductility in the presence of hydrogen [1], is a significant and longstanding problem. It is supported by considerable experimental evidence, however, no consensus has been reached on the dominant underlying mechanism. The two prevailing HE mechanisms for non-hydride forming metals are hydrogen enhanced decohesion (HEDE) and hydrogen enhanced localized plasticity (HELP). The HEDE mechanism assumes that dissolved hydrogen reduces the cohesive strength of the material lattice [2], whereas the HELP mechanism assumes hydrogen promotes dislocation activity (nucleation and or mobility) thereby enhancing plasticity locally

\footnotetext{
${ }^{*}$ Corresponding author

Email addresses: haiyang.yu@materials.ox.ac.uk (Haiyang Yu ), alan.cocks@eng.ox.ac.uk (Alan Cocks), edmund.tarleton@eng.ox.ac.uk (Edmund Tarleton)
}

[3]. It is now accepted that HE is a complex, material and environment dependent process such that no mechanism applies exclusively [4]. For instance, a hydrogen enhanced plasticity-mediated decohesion mechanism was proposed for HE in martensitic steels [5]. This theory claims that hydrogen-enhanced mobility of dislocations leads to local stress build-up and hydrogen concentration elevation close to material interfaces where decohesion is promoted, that is, HELP and HEDE might act in synergy to promote failure.

HE has been investigated both experimentally and numerically. Experimental techniques cover a wide range of spatial and temporal scales, covering the atomistic [6], microscale [7, 8] and macroscale [9]. Hydrogen has been observed to enhance dislocation plasticity, induce global softening and cause premature failure accompanied by a transition from ductile to brittle failure [1]. Modeling techniques used to study HE include analytical calculations, atomistic simulations (MD, DFT) and continuum simulations (FEM). Birnbaum and Sofronis [3] treated hydrogen as a point dilatation and evaluated its influence on the 
stress field of a pair of edge dislocations using linear elasticity theory. They found that hydrogen shields the stress field of an elastic center thereby alleviating the resistance to dislocation motion. This theory is referred to as the "hydrogen shielding effect"or "hydrogen screening effect"which partially accounts for the HELP phenomenon. Over the last decade, atomistic simulations have been widely used for investigating HE. Jiang and Carter [10] performed first principles calculations and found that the ideal fracture energy decreases linearly with increasing hydrogen coverage, supporting the HEDE mechanism. With the same technique, Wang et al. [11, 12] found hydrogen tends to lower the Peierls energy barrier for screw dislocations, increasing their mobility; a similar observation was made by Zhao and $\mathrm{Lu}$ [13] using a multiscale quantum mechanics/molecular mechanics approach. These results support the HELP mechanism. It should be noted that different opinions exist regarding the effect of hydrogen on dislocation mobility. This is likely a consequence of dislocation mobility being determined by the dislocation core structure and so the influence of hydrogen on mobility can depend on the concentration, stress, temperature and material. Song and Curtin [14] observed hydrogen decreased the mobility of edge dislocations in a molecular dynamics (MD) study. The same authors proposed an alternative HE theory, namely hydrogen suppressed dislocation emission, based on their MD results [15]. Assuming the HELP mechanism, the hydrogen effect can be implemented in a conventional failure analysis through a hydrogen softening plasticity law which reduces the yield stress with increasing hydrogen concentration. This idea was introduced by Sofronis et al. [16] who studied hydrogen induced plasticity localisation in a plane strain tensile specimen. Ahn et al. [17] and Liang et al. [18] then studied how hydrogen affects microvoid growth using a unit cell approach. Barrera et al. [19] applied the idea to study hydrogen effects on a notched tensile plate using a coupled mechanical-diffusion analysis. Recently, Yu et al. [20] performed a unit cell analysis and obtained a quantitative description of hydrogen failure loci. In these studies, the hydrogen softening effect is still implemented in a phenomenological manner.

Due to the temporal and spatial gap between atom- istic and continuum scale simulations, a multiscale modelling framework for HE is still lacking. For example, hydrogen enhanced dislocation mobility obtained in atomistic calculations can not be readily translated into the hydrogen softening law as required by continuum plasticity. Discrete dislocation plasticity (DDP) simulations are well suited to bridge this gap as the aggregate behavior of a dislocation ensemble is simulated. In DDP, the motion and interactions of dislocations in a finite volume is simulated by discretising the dislocation network into discrete line segments; allowing the study of dislocation behaviour at the individual dislocation level $[21,22,23]$ and plasticity in micro scale specimens $[24,25,26]$. The key inputs are elastic properties governing dislocation-dislocation interactions and a dislocation mobility law, relating dislocation velocity to the driving force, which can be extracted from atomistic simulations. By incorporating the effects of environmental variables on these properties, DDP can be adapted for investigating the influence of a wide range of environmental factors, such as temperature [27], irradiation [28] and solute concentration [29].

The hydrogen contribution to the stress field was obtained by $\mathrm{Gu}$ and El-Awady [30] by approximating hydrogen atoms as Eshelby inclusions in an isotropic infinite elastic medium; as originally proposed by Cai et al. [31] in 2D. When implemented within dislocation dynamics simulations, the hydrogen elastic shielding effect in an infinite volume was observed [30]. However, the influence of hydrogen in the dislocation core, where linear elasticity breaks down, cannot be accounted for within this linear elastic framework. The influence of hydrogen on the core force is particularly important for bcc metals where the bulk hydrogen concentration is low. To partially account for this Yu et al. [32] implemented a hydrogen dependent dislocation mobility law. In addition the hydrogen image stress was included, enabling simulations of a hydrogen charged microcantilever bend test. Hydrogen enhanced dislocation activity, mechanical softening and enhanced slip planarity were captured at realistic bulk concentrations for bcc materials.

Previous DDP simulations with hydrogen elastic shielding were performed on a single bcc slip sys- 
tem [32]. Although hydrogen was found to enhance plasticity by promoting dislocation activity, the dislocation stress counteracts the applied stress, as this lowers the elastic energy of the beam. Consequently, in the absence of obstacles (so that dislocations are free to move in response to a driving force) hydrogen enhanced plasticity reduces the stress, indicating that stress-controlled failure would be less likely.

In real engineering materials the motion of dislocations is impeded by obstacles. For example dislocation pile-ups can form against strong obstacles preventing dislocations from reaching lower energy positions. This mechanism can build up a large dislocation array generating a stress field that enhances the applied stress field to induce failure. This provides a possible $\mathrm{HE}$ mechanism, as hydrogen can increase the dislocation density in a pile-up, further raising the stress. Therefore, considering obstacles in DDP simulations is essential to simulate hydrogen embrittlement. In this work we simulate a single slip system containing an obstacle near a free surface to prevent dislocations from exiting. A hydrogen enhanced stress concentration develops which hints at a possible precursor to HE. In real materials, obstacles can be of various types, ranging from inclusions, or precipitates, to strong dislocation locks. Here, it is approximated as a "super dislocation".

The hydrogen elastic shielding stress is very weak $[32,33]$, therefore, extremely high concentrations must be simulated to observe a noticeable effect. In contrast, the effect of hydrogen on the dislocation core region is significant as hydrogen alters the dislocation core energy profile. This idea was recently developed by Sills and Cai [34] for fcc materials. Following their approach, we calibrated the hydrogen core energy over a range of realistic concentrations and implemented it in DDP. To our knowledge a hydrogen dependent fcc mobility law is not yet available in the literature and so was neglected in this study.

\section{Hydrogen DDD simulation method}

The details of the model employed here have been presented in $[32,35]$. Here we provide a brief overview of the approach. Dislocations are represented by discrete straight segments. The nodal force $\boldsymbol{F}^{k}$ of node $k$ at position $\boldsymbol{X}^{k}$ is evaluated in each time increment for every node, and the dislocation structure is updated using a nodal velocity $\boldsymbol{V}^{k}$ determined through an fcc mobility law.

\subsection{Nodal forces}

The nodal force $\boldsymbol{F}$ has contributions from the segmentsegment interaction force obtained from the non-singular dislocation stress field and the dislocation core force. Both of which are reduced or shielded by hydrogen, and represented by two additional forces, the hydrogen core force and the elastic hydrogen force. In addition there are forces due to the corrective (image and applied) stress fields. Therefore, the total nodal force consists of five terms:

$$
\begin{aligned}
\boldsymbol{F}^{k} & =\sum_{l} \sum_{i, j} \tilde{\boldsymbol{f}}_{i j}^{k l}\left(\boldsymbol{X}^{k}\right)+\sum_{l} \sum_{i, j} \overline{\boldsymbol{f}}_{i j}^{k l}\left(\boldsymbol{X}^{k}\right) \\
& +\sum_{l} \boldsymbol{f}_{c}^{k l}\left(\boldsymbol{X}^{k}\right)+\sum_{l} \overline{\boldsymbol{f}}_{c}^{k l}\left(\boldsymbol{X}^{k}\right)+\sum_{l} \hat{\boldsymbol{f}}^{k l}\left(\boldsymbol{X}^{k}\right) \\
& =\tilde{\boldsymbol{F}}^{k}+\overline{\boldsymbol{F}}^{k}+\boldsymbol{F}_{c}^{k}+\overline{\boldsymbol{F}}_{c}^{k}+\hat{\boldsymbol{F}}^{k},
\end{aligned}
$$

where $\tilde{\boldsymbol{f}}_{i j}^{k l}\left(\boldsymbol{X}^{k}\right)$ is the interaction force at node $k$, due to segment $i \rightarrow j$ integrated along segment $k \rightarrow l$; this is summed over all segments $i \rightarrow j$ inside the domain, including the self force due to segment $k \rightarrow l$, this is then summed over all nodes $l$ which are connected to node $k$ to give the interaction force on node $k, \tilde{\boldsymbol{F}}^{k}$. Similarly, $\overline{\boldsymbol{F}}^{k}$ is the hydrogen elastic force evaluated at node $k . \boldsymbol{F}_{c}^{k}$ is the dislocation core force, $\overline{\boldsymbol{F}}_{c}^{k}$ is the hydrogen core force shielding term (due to hydrogen reducing the magnitude of the dislocation core energy) and $\hat{\boldsymbol{F}}^{k}$ is the corrective elastic force evaluated with the finite element method using superposition [36] to account for the finite boundary.

The hydrogen elastic shielding effect is accounted for through $\overline{\boldsymbol{F}}^{k}$, which was derived in [30] by approximating hydrogen atoms as Eshelby inclusions. This term does not account for the interaction between hydrogen atoms. According to Sills and Cai [34], the solute-solute interactions can give rise to a significant change in near-core energy in the presence of hydrogen. This change in core energy due to solutesolute interactions constitutes the hydrogen core effect which is missing in the hydrogen elastic shielding theory. 
The change in dislocation core energy due to hydrogen is denoted $E_{c}^{H}$, so in the presence of hydrogen, the dislocation core energy becomes

$$
E_{c}^{e f f}=E_{c}+E_{c}^{H},
$$

with $E_{c}$ being the usual (hydrogen free) core energy. Both $E_{c}$ and $E_{c}^{H}$ are dependent on the dislocation character. For edge segments $E_{c}^{H}<0$ whereas $E_{c}^{H}=$ 0 for screw segments; so hydrogen reduces the core energy of dislocations with an edge component and has no effect on pure screw dislocations. The expression for the dislocation core force in DDD, on the segment connecting nodes $k \rightarrow l$, is [35]

$$
\boldsymbol{f}_{c}^{k l}=-E_{c}(\theta) \boldsymbol{l}^{k l}+\frac{d E_{c}(\theta)}{d \theta}\left(\frac{\boldsymbol{b}_{e}^{k l}}{\left|\boldsymbol{b}_{e}^{k l}\right|}\right),
$$

where $\theta$ is the angle between the line direction $\boldsymbol{l}^{k l}$ and Burgers vector of the segment and the edge component of the Burgers vector is $\boldsymbol{b}_{e}^{k l}$. The first term on the right hand side acts along the line direction to shrink the segment, while the second term is a moment tending to rotate the segment to the orientation with lowest core energy; in the absence of hydrogen this is the screw orientation $(\theta=0)$. In the presence of hydrogen the total core force becomes

$$
\boldsymbol{f}_{c}^{k l}+\overline{\boldsymbol{f}}_{c}^{k l}=-E_{c}^{\mathrm{eff}}(\theta) \boldsymbol{l}^{k l}+\frac{d E_{c}^{\mathrm{eff}}(\theta)}{d \theta}\left(\frac{\boldsymbol{b}_{e}^{k l}}{\left|\boldsymbol{b}_{e}^{k l}\right|}\right)
$$

with

$$
\overline{\boldsymbol{f}}_{c}^{k l}=-E_{c}^{H}(\theta) \boldsymbol{l}^{k l}+\frac{d E_{c}^{H}(\theta)}{d \theta}\left(\frac{\boldsymbol{b}_{e}^{k l}}{\left|\boldsymbol{b}_{e}^{k l}\right|}\right) .
$$

As $E_{c}^{\mathrm{eff}}<E_{c}$, hydrogen reduces the magnitude of the core force; which we refer to as hydrogen core force shielding.

The approach for calibrating the hydrogen core energy has been established by Sills and Cai [34]

$$
E_{c}^{H}=\Delta G-\Delta G_{e l},
$$

where $\Delta G$ is the total free energy change due to hydrogen and $\Delta G_{e l}$ the part due to hydrogen elastic shielding. Following this approach and adopting the same parameters as in [34], we calibrated hydrogen core energies at room temperature over a range of concentrations realistic for fcc metals: $c_{0} \in(0,1000)$ appm. The results are presented in Fig. 1. As shown

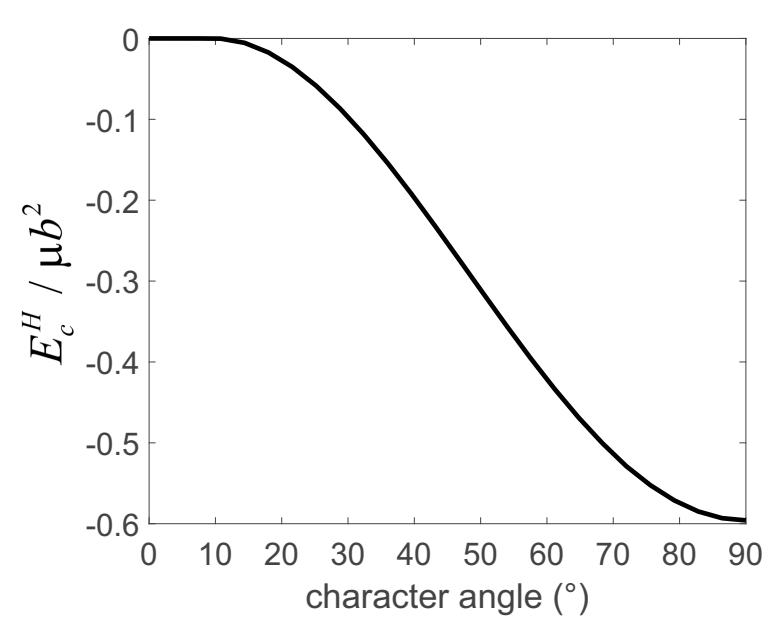

(a)

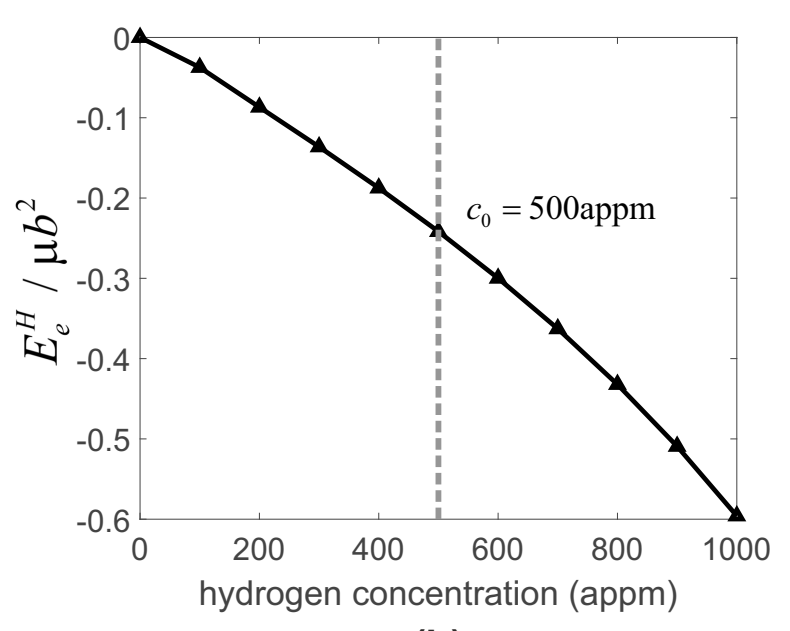

(b)

Fig. 1 (a) The variation of hydrogen core energy with respect to the character angle, calibrated with $c_{0}=1000$ appm. (b) The variation of hydrogen core energy over the range of concentrations $c_{0} \in(0,1000)$ appm, calibrated for an edge dislocation.

in Fig. 1(a), the hydrogen core energy has an approximately sinusoidal dependence on $\theta[34,37]$. A screw dislocation $\left(\theta=0^{\circ}\right)$ has no pressure field, so its free energy is unchanged by hydrogen. The hydrogen core energies due to an edge dislocation $(\theta=$ $90^{\circ}$ ) over a range of concentrations are plotted in Fig. 1(b). The hydrogen core energy of a mixed dislocation is determined by interpolation between these two cases,

$$
E_{c}^{H}\left(\theta, c_{0}\right)=\sin ^{2}(\theta) E_{e}^{H}\left(c_{0}\right),
$$


where $E_{e}^{H}$ is the hydrogen core binding energy of an edge dislocation calibrated in Fig. 1(b).

\subsection{Dislocation mobility law}

For each segment $k l$ with length $L^{k l}$, a drag tensor $\boldsymbol{B}^{k l}$ is determined according to the segment character, the nodal velocity $\boldsymbol{V}^{k}$ at node $k$ is then obtained using

$$
\left[\frac{1}{2} \sum_{l} L^{k l} \boldsymbol{B}^{k l}\right]^{-1} \boldsymbol{F}^{k}=\boldsymbol{V}^{k},
$$

where the sum is again over all nodes $l$ connected to node $k$, and $\boldsymbol{F}^{k}$ is the nodal force determined by Equation 1.

As discussed in [38], all dislocation segments are constrained to glide in well defined slip planes in fcc, and their drag tensor can therefore be expressed as

$$
\boldsymbol{B}^{k l}\left(\boldsymbol{l}^{k l}\right)=B_{g}\left(\boldsymbol{m}^{k l} \otimes \boldsymbol{m}^{k l}\right)+B_{c}\left(\boldsymbol{n}^{k l} \otimes \boldsymbol{n}^{k l}\right)+B_{l}\left(\boldsymbol{l}^{k l} \otimes \boldsymbol{l}^{k l}\right)
$$

where $B_{g}, B_{c}$ and $B_{l}$ are the drag coefficients for glide, climb and motion along the line direction, respectively. The unit vectors are the line direction $\boldsymbol{l}^{k l}$, the slip plane normal $\boldsymbol{n}^{k l}$ and glide direction $\boldsymbol{m}^{k l}$. In this work only glide motion is allowed, which is achieved by assigning $B_{c} \gg B_{g} \gg B_{l}$ for all segments. The same glide coefficient $B_{g}=5 \times 10^{-4} \mathrm{~Pa}$ s was used for all segments; so that edge and screw segments have the same mobility.

\subsection{Material parameters}

Here we investigate the effect of hydrogen on dislocation activity rather than simulating experimental data, where additional mechanisms are likely active. To be consistent with [34], nickel was selected as the model system, and the same parameters were used. These were: the atomic volume for nickel, $\Omega=10.77 \AA^{3}$, the lattice parameter, $a_{0}=3.524 \AA$, Burgers vector magnitude, $b=2.49 \AA$, shear modulus, $\mu=134.3 \mathrm{GPa}$, and Poisson's ratio $v=0.201$. The cut-off core radius used in the DDD simulation was $r_{c}=4.3 b$.

An initial uniform bulk hydrogen concentration of 500 appm was applied, and assumed to redistribute instantaneously in chemical equilibrium with the evolving stress field during the simulation [34]. This assumption is often made for fcc materials [39, 40].
This is a reasonable approximation if the loading rate is low, and means the results should be viewed as an upper bound on the effect of hydrogen [34]. As the relative speed between dislocation and hydrogen increases, hydrogen lags behind and has less influence. The approximation at the other extreme where hydrogen is regarded as stationary is examined in [30].

\section{The effect of hydrogen on a Frank-Read source}

It is useful to first analyse the effect of hydrogen on the operation of a Frank-Read (FR) source. The insight gained can be used to help explain the behaviour of the micro-cantilever beam reported in section 4. A FR source on the $\frac{1}{2}[10 \overline{1}](111)$ slip system in an infinite medium was simulated. An edge FR source is shown in Fig. 2 as an example. It is pinned at both ends, with an initial line direction $l=[\overline{1} 2 \overline{1}]$ and length $l_{0}$. A pure shear with magnitude $\tau$ was applied out-of-plane

$$
\boldsymbol{\sigma}=\boldsymbol{R}^{T}\left[\begin{array}{ccc}
0 & 0 & \tau \\
0 & 0 & 0 \\
\tau & 0 & 0
\end{array}\right] \boldsymbol{R}
$$

where $\boldsymbol{R}$ is the rotation matrix which transforms a vector defined in the crystal coordinate system (aligned with the model) to the dislocation coordinate system defined by $\boldsymbol{b}, \boldsymbol{l}$ and $\boldsymbol{n}$

$$
\boldsymbol{R}=\left[\begin{array}{ccc}
1 / \sqrt{2} & 0 & -1 / \sqrt{2} \\
-1 / \sqrt{6} & 2 / \sqrt{6} & -1 / \sqrt{6} \\
1 / \sqrt{3} & 1 / \sqrt{3} & 1 / \sqrt{3}
\end{array}\right]
$$

where the rows of $\boldsymbol{R}$ are the unit vectors along $\boldsymbol{b}$, $\boldsymbol{l}$ and $\boldsymbol{n}$. We define $\boldsymbol{b}$ using the RH/FS convention [38] so the initial edge segment has an extra half plane below the slip plane which moves to the left under a positive shear when viewed from above as in Fig. 2. When the magnitude of the applied stress is sufficiently high, the FR source generates dislocation loops as illustrated in Fig. 2.

As the applied stress increases, the FR source will bow out and form a semi-circle, if the stress is sufficient to further bow out the dislocation line then the source can expand freely, as doing so reduces the curvature. Here we define the nucleation stress, or the strength of the source $\tau_{c}$, as the stress 


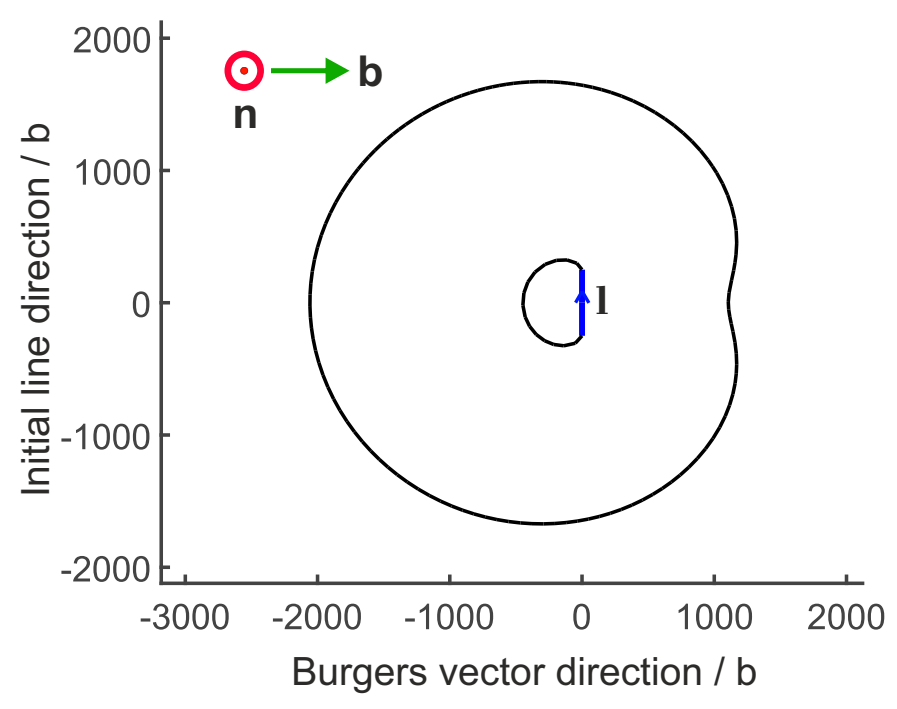

Fig. 2 Illustration of the edge type FR source, the slip plane normal points out of the page under an applied shear stress.

required to bow out beyond a semi-circle. We define this as when the angle between $\boldsymbol{l}$ at the pinning points and the initial line direction of the source first reaches $\pi / 2$. It is noted that nucleation was defined in [41] as when the total line length exceeds $m l_{0}$, with $m \geq 2$. The semi-circle condition used here corresponds to $m=\pi / 2 \approx 1.57$. As a semi-circle expands freely, these two criteria are equivalent, given sufficient simulation time. We verified this and found that these methods give similar predictions to the activation stress in approximate agreement with the theoretical value $\tau_{c}=\alpha \mu b / L$ for screw and edge FR sources [42].

To evaluate how hydrogen affects dislocation generation, we considered two effects independently: the hydrogen elastic shielding, $\bar{f}$, and hydrogen core force shielding, $\overline{\boldsymbol{f}}^{c}$ in Equation 1. The resulting activation stress as a function of initial source length considering each hydrogen shielding force individually are compared to the hydrogen free case for an edge and screw source in Fig. 3. The hydrogen-free nucleation stress is higher for the screw type source than for the edge type, as expected from dislocation theory [42]. Hydrogen core force shielding reduces the strength of the screw source, promoting the nucleation of dislocation loops, at small concentrations typically found in fcc materials, but has no effect on the edge source. In contrast, the influence of

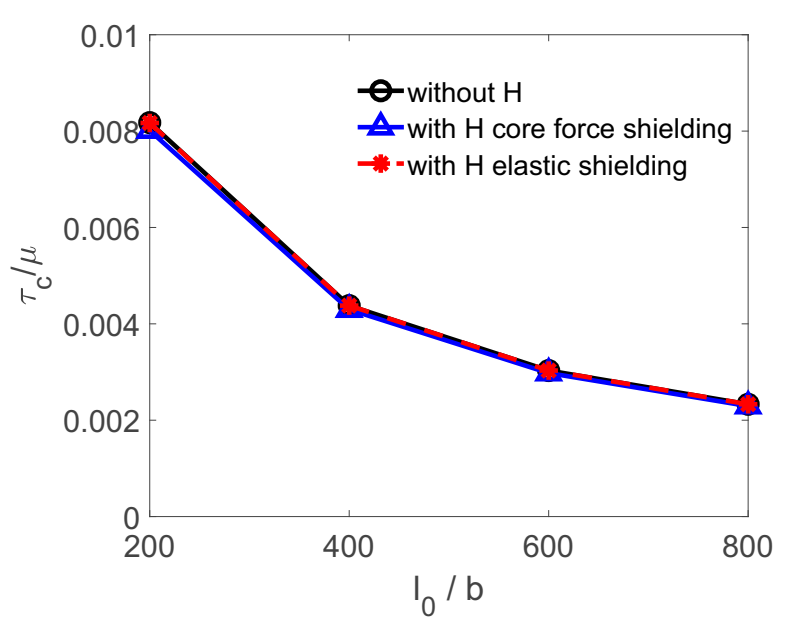

(a)

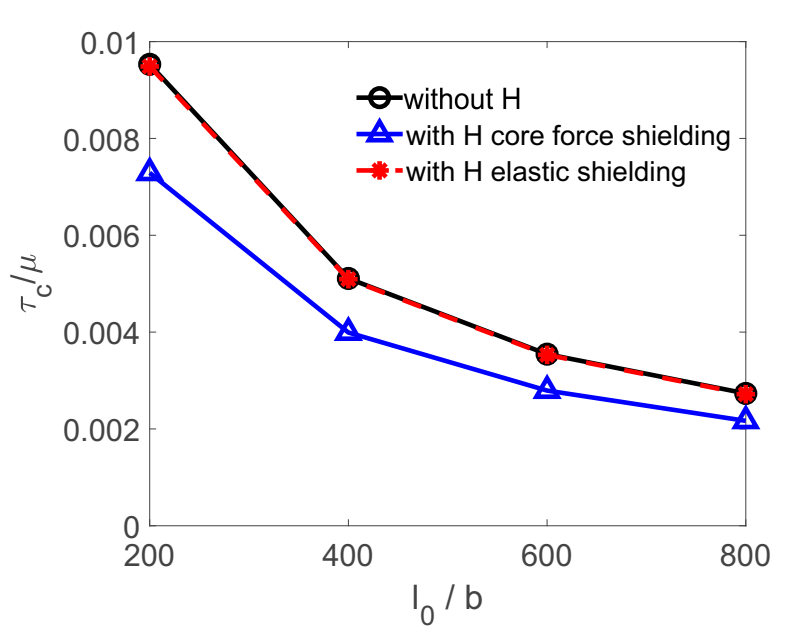

(b)

Fig. 3 The FR activations stresses for (a) an edge type source and (b) a screw type source. In both cases, the hydrogen concentration is 500 appm, realistic for fcc materials.

hydrogen elastic shielding is negligible at this concentration. Further, we performed additional simulations with hydrogen elastic shielding and found that it could also reduce the nucleation stress, but the applied concentration had to be unrealistically high, $\geq 1 \times 10^{5} \mathrm{appm}$. Such evidence strongly indicates that the influence of hydrogen elastic shielding is negligible for fcc materials, as previously noted for bcc materials [32].

Sills and Cai [34] also observed that Hydrogen does not affect the nucleation stress for an edge source and explained it from the perspective of line energy. Here we provide a rationale based on direct analysis 
of the nodal forces. As indicated in Equation 4, the dislocation core force tends to shrink a dislocation segment and rotate it to a lower energy (screw) configuration. Since the hydrogen core energy is negative and lower for edge than screw, as in Fig. 1(a), the hydrogen core force has the opposite effect to the usual core force, acting to stretch the dislocation line and rotate it to an edge orientation.

Equation 6 enables us to work out the direction of the hydrogen core force. Suppose an initially pure edge dislocation (FR source), as shown in Fig. 4(a), bows out slightly by an angle $\alpha=\pi / 2-\theta$, the force on node 2 results from 4 components: the line tensions $f_{l}^{12}$ and $f_{l}^{23}$, and the rotation forces $f_{b_{e}}^{12}$ and $f_{b_{e}}^{23}$ perpendicular to the line segments. The rotation forces tend to facilitate the bowing while the line tensions impede it. On the contrary, the rotation part of the hydrogen core force $\bar{f}_{b_{e}}^{12}$ and $\bar{f}_{b_{e}}^{23}$ tends to impede the bowing out while the line tension part $\bar{f}_{l}^{12}$ and $\bar{f}_{l}^{23}$ facilitates it. For the screw case, shown in Fig. 4(b), all components of the core force act to impede the bowing, so hydrogen reduces the nucleation stress of a screw source. This also explains the higher activation stress for the screw source in the absence of hydrogen. Substituting the interpolation scheme in Equation 8 into Equation 6, the total hydrogen core force on node 2 in Fig. 4 (a) and (b) is, respectively

$$
\begin{aligned}
\overline{\boldsymbol{F}}_{c}^{2} & =2 \cdot \sin \alpha \cos ^{2} \alpha\left|E_{e}^{H}\right| \frac{l_{0}}{2} \hat{\boldsymbol{e}}_{1} \\
& \approx \alpha\left|E_{e}^{H}\right| l_{0} \hat{\boldsymbol{e}}_{1}, \quad \text { (a) near edge; } \\
\overline{\boldsymbol{F}}_{c}^{2} & =2 \cdot\left(\sin ^{3} \theta+2 \sin \theta \cos ^{2} \theta\right)\left|E_{e}^{H}\right| \frac{l_{0}}{2} \hat{\boldsymbol{e}}_{2} \\
& \approx 2 \theta\left|E_{e}^{H}\right| l_{0} \hat{\boldsymbol{e}}_{2}, \quad \text { (b) near screw. }
\end{aligned}
$$

Given a small perturbation, the hydrogen nodal force on a near screw segment is about twice as large as for a near edge segment and the ratio grows as the angle increases. This explains why a more pronounced hydrogen effect is observed in the screw case.

Although hydrogen core force shielding has no influence on the initial activation of an edge source, hydrogen was found to have a considerable influence during the expansion of the nucleated loops. An edge source of length $l_{0}=800 \mathrm{~b}$ under an applied shear stress of $\tau=500 \mathrm{MPa}$, with and without hydrogen core force shielding at $c_{0}=500$ appm are superim- (a)
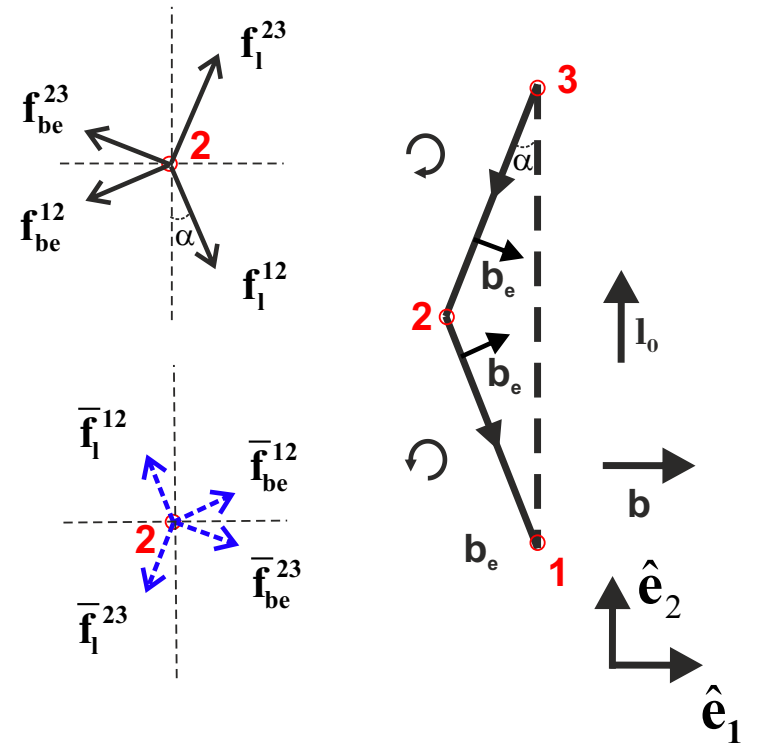

(b)

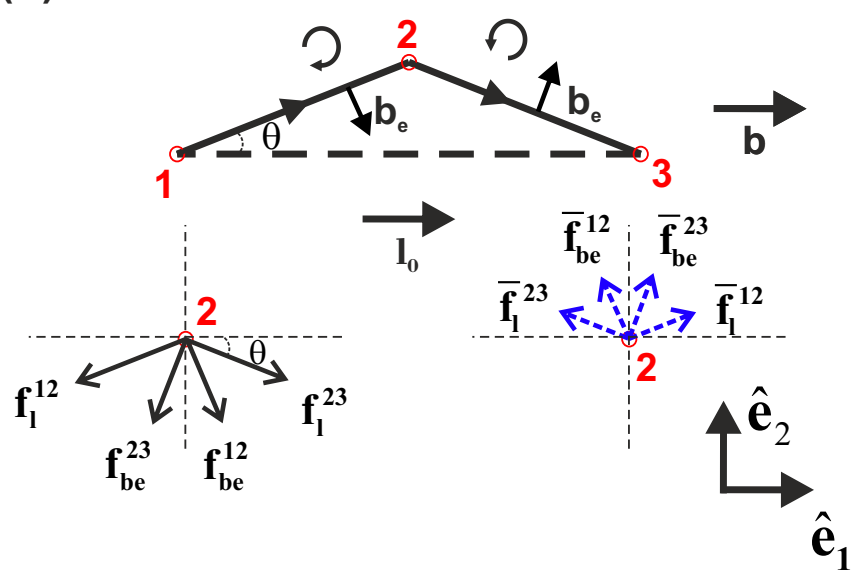

Fig. 4 The directions of the core force and hydrogen core force using Equation 4 and Equation 6. (a) In the case with an initially pure edge segment (FR source), hydrogen exerts a back force preventing the segment from bowing out. (b) In the pure screw case, the hydrogen core force always facilitates the bowing of the segment.

posed in Fig. 5 after a simulated time $t=36 \mathrm{~ns}$. One additional dislocation loop was generated in the presence of hydrogen. This should be attributed to the effect of hydrogen on the expansion of the generated loops. The edge segments have a reduced spacing due to hydrogen. The loops are slightly contracted along the Burgers vector direction when hydrogen is present. This helps explain the reduced spacing in an edge dislocation pileup. To understand this the nodal forces are decomposed into hydrogen and nonhydrogen parts in Fig. 6. 


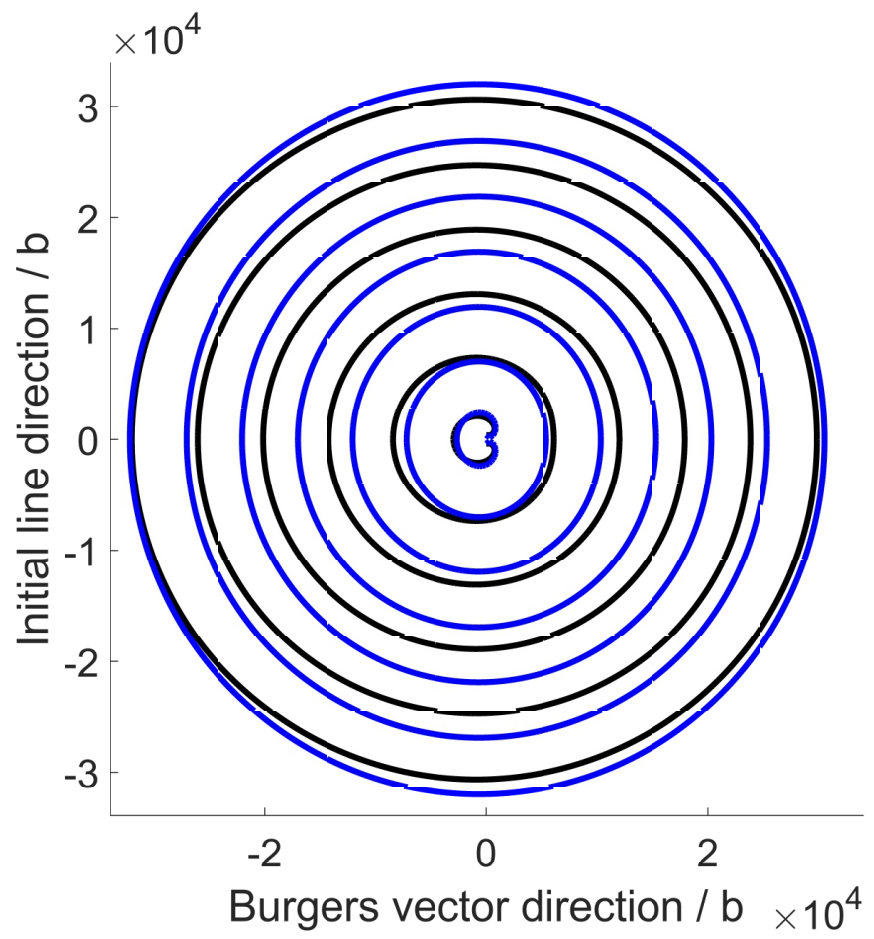

Fig. 5 Dislocation loops generated by the edge FR source after $36 \mathrm{~ns}$ with $c_{0}=500$ appm hydrogen (blue) and without hydrogen (black). An additional loop has been nucleated due to hydrogen.

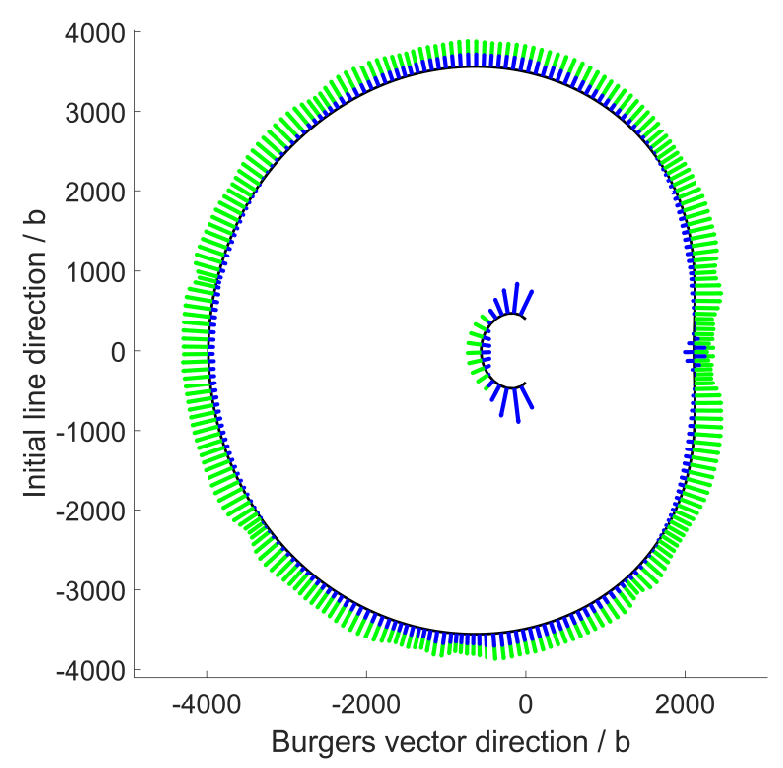

Fig. 6 The nodal force at a simulated time of $5.7 \mathrm{~ns}$ $\tilde{\boldsymbol{F}}+\boldsymbol{F}^{c}+\hat{\boldsymbol{F}}$ due to the dislocation interaction and applied stress are represented by green arrows and hydrogen core shielding force $\overline{\boldsymbol{F}}^{c}$ blue. Note $\overline{\boldsymbol{F}}^{c}$ force arrows are scaled by $20 \times$ in order to make them visible.
When the FR source bows out, the hydrogen core shielding force on the edge type segments are much smaller, and in opposition to, the elastic forces, whereas they act together to accelerate the screw and mixed segments. Hydrogen therefore promotes the expansion of the dislocation loops generated from the FR source. For a screw source, similar behaviour was also observed.

The nucleation time is a required input parameter for 2D DDP simulations [43]. Knowing the effects of hydrogen on activation stress and nucleation time is therefore useful for simulating hydrogen with 2D DDP. Fig. 5 indicates the average nucleation time is reduced due to hydrogen. We define the nucleation time as when the first dislocation loop is nucleated by the FR source, which can be extracted from the simulations by detecting the first annihilation event between the dislocation segments that have bowed back. The simulations were performed on the same edge FR source of length $l_{0}=800 \mathrm{~b}$ and over a range of applied stresses $350-700 \mathrm{MPa}$, with $350 \mathrm{MPa}$ being approximately the nucleation stress. The results are presented in Fig. 7, including the cases without hydrogen, with hydrogen elastic shielding and with hydrogen core force shielding. After the FR source is activated, i.e. the critical con-

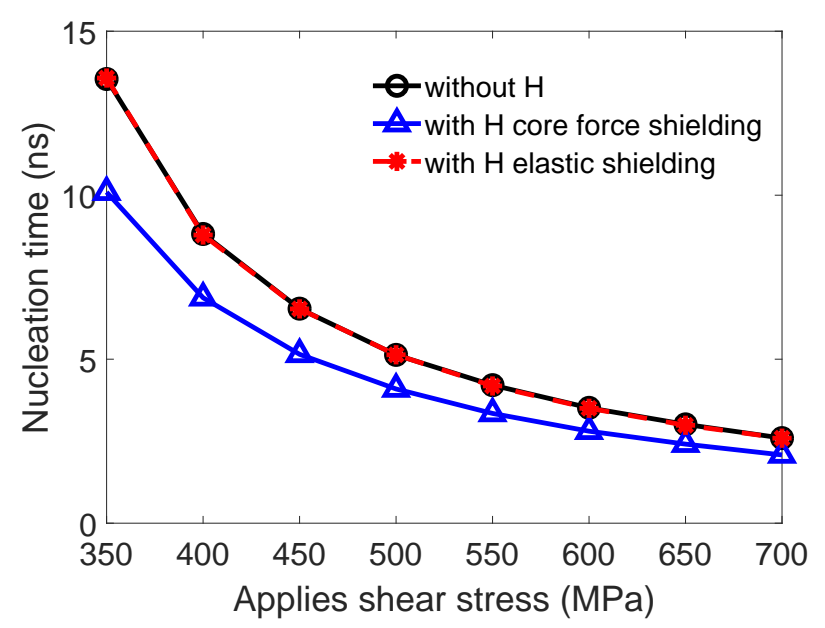

Fig. 7 The time to nucleated the first dislocation loop from the edge FR source with length $l_{0}=800 b$.

figuration is reached, the dislocation line will keep expanding until nucleating the first loop, hence the nucleation time is controlled by the speed of dislocation expansion. Therefore, the nucleation time de- 
creases with applied stress. The nucleation time is reduced when hydrogen is present, because hydrogen tends to promote the expansion of the dislocation loops, as discussed earlier. The nucleation time is dependent on the length of the source, the applied stress and the drag coefficients. It should, therefore, ideally be calibrated for a given material. Hydrogen elastic shielding does not produce any change in the nucleation time whereas at low stresses, hydrogen core force shielding reduces the nucleation time by $\sim 30 \%$.

\section{A hydrogen charged microcantilever}

An end loaded microcantilever with dimensions $L_{x}=15 \mu \mathrm{m}$ and $L_{y}=L_{z}=2.5 \mu \mathrm{m}$ was simulated to investigate the effect of hydrogen in a finite volume. For simplicity, only the $\frac{1}{2}[10 \overline{1}](111)$ slip system was considered, and the crystal was rotated such that the $\boldsymbol{n} \times \boldsymbol{b}$ direction was parallel to the $y$ axis in the model, as shown in Fig. 8. The rotation is performed around the $\left[w_{x}, w_{y}, w_{z}\right]=\frac{1}{\sqrt{2}}[1,0,-1]$ axis for an angle $\theta=$ 0.6155 , and the rotation matrix is obtained following Rodrigues' rotation formula

$$
\boldsymbol{Q}=\boldsymbol{I}+\sin (\theta) \boldsymbol{W}+(1-\cos (\theta)) \boldsymbol{W}^{2},
$$

with

$$
\boldsymbol{W}=\left[\begin{array}{ccc}
0 & -w_{z} & w_{y} \\
w_{z} & 0 & -w_{x} \\
-w_{y} & w_{x} & 0
\end{array}\right]
$$

With this configuration, the line direction of the dislocation pile-up was parallel to the $y$ direction in the model so that $\boldsymbol{l} \rightarrow$ [010] and both $\boldsymbol{b} \rightarrow$ [101] (which is unchanged) and $\boldsymbol{n} \rightarrow$ [101] are in the $x z$ plane of the model. The initial dislocation structure consisted of ten identical square prismatic loops with eight nodes, 6 of which were pinned, as shown in the Fig. 8 inset. Under an applied stress, each prismatic loop can act as pairs of FR sources. The boundary conditions were: $\boldsymbol{u}(0, y, z)=[0,0,0], \boldsymbol{u}\left(L_{x}, y, L_{z}\right)=$ $[0,0, U]$ with an applied displacement of $U=-0.3 \mu \mathrm{m}$ and the remaining surfaces were traction free. Fully integrated 8 node linear brick finite elements, $0.3 \mu \mathrm{m}$ in size, were used to solve for the corrective elastic solution at every time increment. The simulated hydrogen concentration was 500 appm.

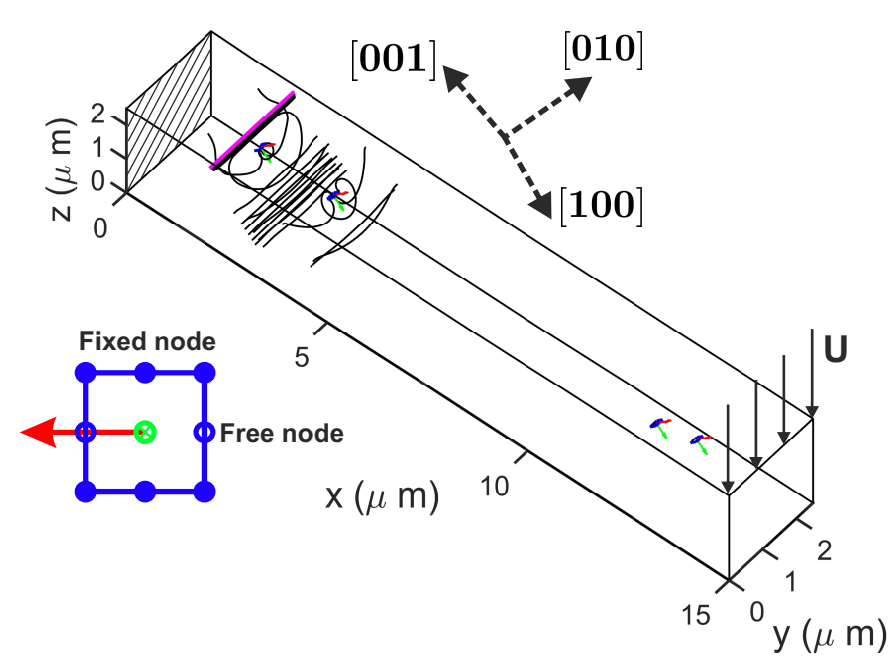

Fig. 8 Square prismatic loops with two free nodes are randomly generated on the $\frac{1}{2}[10 \overline{1}](111)$ slip system as initial dislocation sources. The source (blue line), its Burgers vector (green arrow) and normal (red arrow) are shown. The crystal is rotated around the $\left(w_{x}, w_{y}, w_{z}\right)=$ $\frac{1}{\sqrt{2}}(1,0,-1)$ axis for an angle $\theta=0.6155$, placing both the slip plane normal and Burgers vector inside the global $x z$ plane. An obstacle (the pinned magenta dislocation) is also shown.

When loaded, each prismatic loop acts as a pair of FR sources generating dislocation loops continuously. The majority of the segments exit at the free surfaces with only edge segments (with an extra half plane above the slip plane) remaining, which are trapped in the low stress region in the centre of the beam. To form a dislocation pile-up in regions other than the neutral axis, obstacles for dislocation motion have to be introduced. In subsection 4.2 a pinned super dislocation with a Burgers vector of magnitude $5 b$ and line direction $\boldsymbol{l}_{O B}=[0,1,0]$ was used to prevent dislocations exiting at the upper free surface.

The initial dislocation structure used here was chosen to provide a clear demonstration of the hydrogen core force shielding effect. It includes only a single slip system and allows well organized dislocation pile-ups to form. With this idealised simulation we are not aiming to reproduce any experimental data but simply to examine how hydrogen might promote the development of high local stresses.

\subsection{Hydrogen core force shielding}

Identical simulations were performed with and without hydrogen. The dislocation structures obtained 
at the same applied displacement $U=-0.3 \mu \mathrm{m}$ are superimposed in Fig. 9. With hydrogen core force shielding, more dislocations were generated, as shown in Fig. 10 whereas the hydrogen elastic shielding effect was negligible. Hydrogen increased the dislo-

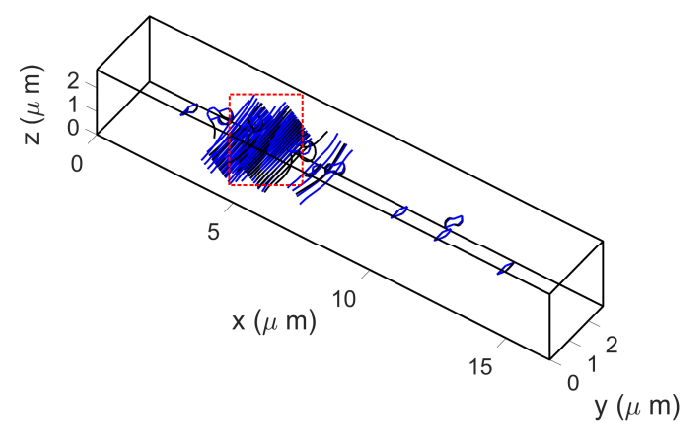

(a)

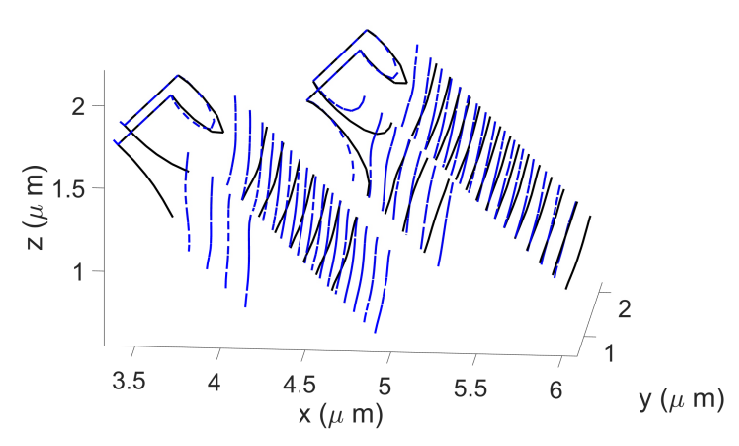

(b)

Fig. 9 (a) Superposition of two simulations both without an obstacle but with hydrogen core fore shielding (blue) and without hydrogen (black). (b) Zooming-in on two pile-ups (dashed red box) in (a) shows hydrogen increases the number of dislocations in a pile up.

cation density in the pile-ups by reducing the dislocation spacing, as shown in Fig. 9(b). This is due to hydrogen core force shielding which accelerates the expansion of the loops and flattens the loop in the burgers vector direction. Globally, hydrogen is observed to induce earlier plastic yielding and soften the flow behavior, as shown in Fig. 10(b), which is consistent with the HELP mechanism [44]. Note that any changes in the results with the addition of hydrogen elastic shielding were negligible. Therefore, hydrogen core force shielding appears to be the mechanism driving HELP. In recent nanoindentation tests, Wang et al. [45] observed that hydrogen decreased the initial pop-in stress. This is consistent with the simulations presented here, where hydrogen is seen

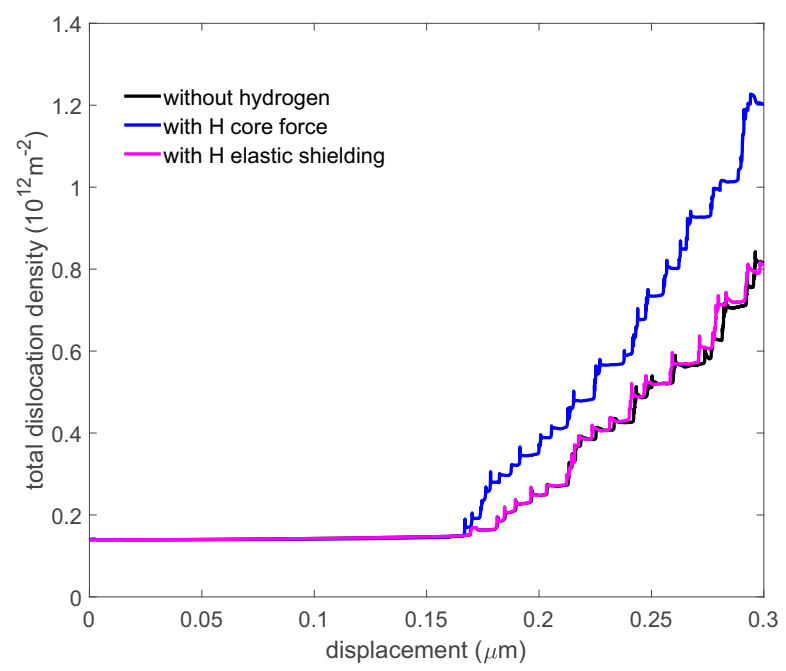

(a)

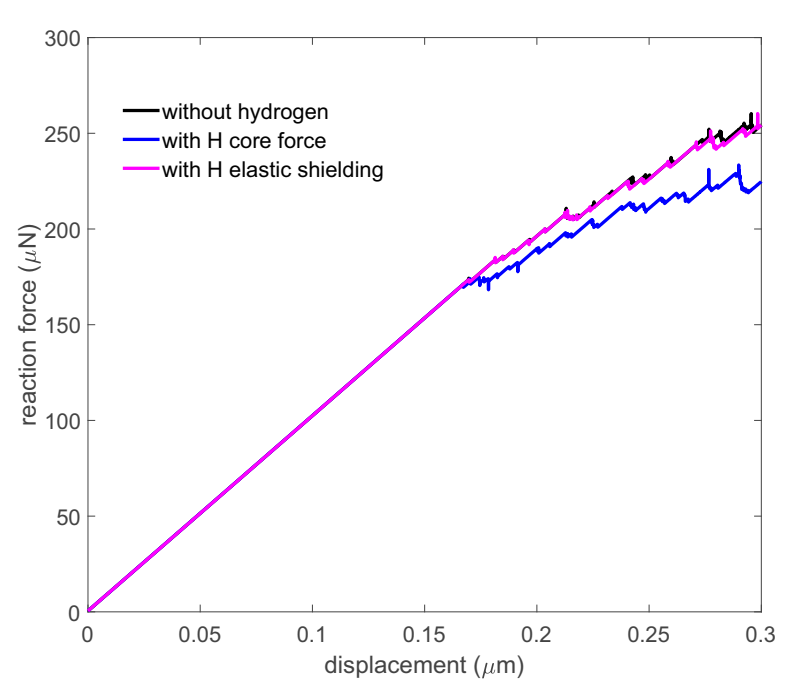

(b)

Fig. 10 (a) The evolution of total dislocation density and (b) the load-displacement curves with hydrogen core force shielding (blue), hydrogen elastic shielding (magenta) and without hydrogen (black). Hydrogen increases dislocation density and softens the global flow behavior; obtained with $c_{0}=500 \mathrm{appm}$.

to lower the initial yield stress as shown in Fig. 10(b).

The stress field with and without hydrogen are shown in Fig. 11. Both the corrective elastic field (due to the applied bending and image stresses) and the dislocation stress are shown. With more dislocations generated, the dislocation stress is higher in the presence of hydrogen, as shown in Fig. 11 (a) and (b). However, the dislocation stress field is compressive above the neutral plane and tensile below, coun- 


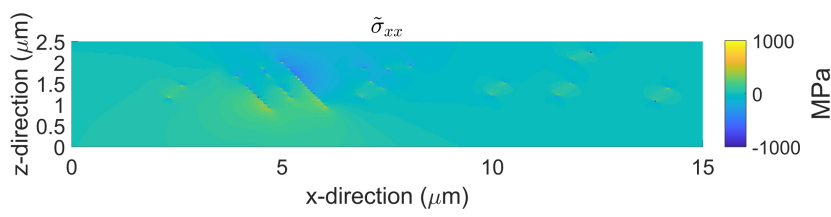

(a)

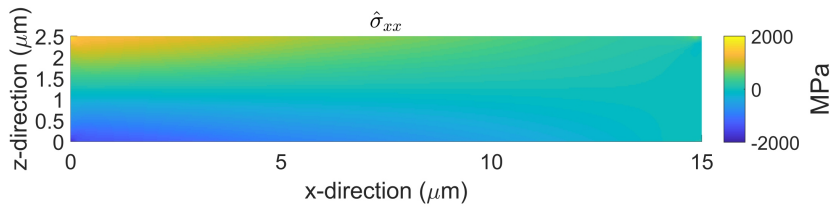

(c)

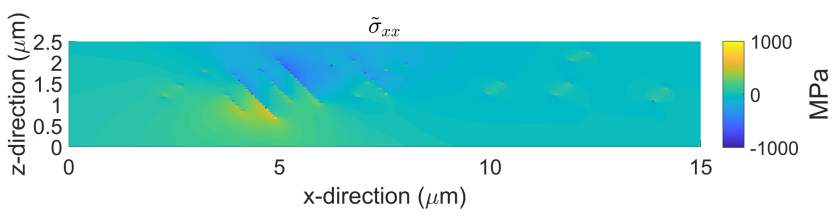

(b)

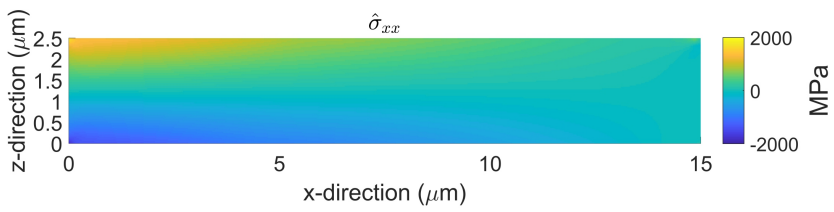

(d)

Fig. 11 (a) The contributions to the axial stress field without any obstacles from dislocations $\tilde{\sigma}_{x x}$ in the absence of hydrogen and (b) with hydrogen; (c) The corrective stress field $\hat{\sigma}_{x x}$ required to satisfy the boundary conditions without hydrogen and (d) with hydrogen.

teracting the corrective field, as previously noted in the literature [24, 43]. Hydrogen therefore lowers the total stress field as the increased plasticity relaxes the system. This is also why softening occurs in the stress-strain (load-displacement) response. Therefore, in the absence of any obstacles, hydrogen is not expected to induce premature failure, if we assume failure is stress controlled. Any dislocations which raise the stress will be attracted to the free surface and exit in order to lower the elastic energy of the system. In reality, obstacles to dislocation motion can prevent dislocations leaving. If an obstacle generates a pileup of the opposite type then hydrogen will increase the stress at the head of the pile-up promoting failure.

\subsection{Hydrogen induced stress elevation at an obsta- cle}

As a first step towards studying the role of hydrogen in promoting failure (HE) a line obstacle parallel to the $y$ axis was simulated, equivalent to a point obstacle in a plane strain two dimensional simulation [43]. The obstacle is simply a pinned dislocation segment near the upper free surface which prevents the mobile dislocations from exiting, as shown in Fig. 8. The obstacle then generates an additional edge dislocation pile-up. It should be noted that this treatment is just a simplification, in reality the obstacle strain field and geometry should be considered or multiple slip systems could be simulated to generate sessile dislocation junctions. The purpose here is to highlight the importance of obstacles in hydrogen induced failure. While keeping the same initial dislocation structure and boundary conditions, simulations were performed with and without hydrogen. The resulting dislocation structures are superimposed in Fig. 12. A dislocation pile-up is formed against the dislocation obstacle which is close to the upper free surface. Fig. 12(b) shows hydrogen also increases the dislocation density in the pile-up formed due to the obstacle. This dislocation pile-up generates a tensile stress field above the slip plane which increases the applied tensile field and so raises the total stress, as shown in Fig. 13(a). Therefore, a stress hot spot is observed in the total stress contour shown in Fig. 13(c), which might cause material failure. It should be noted that the stress generated by the obstacle ("super dislocation") has been excluded in the plots, so the stress hot spot is only due to the dislocations that are generated. By increasing the number of dislocations piling up against the obstacle, the dislocation stress field generated around the obstacle is elevated, leading to a more severe stress concentration in the total stress contour; this is illustrated more clearly by zooming in on the obstacle, as shown in Fig. 14.

This mechanism can apply to a variety of scenarios, for instance, the case where dislocations pile up against dislocation junctions or defects and the case where dislocations are stopped by grain boundaries. In all these cases hydrogen will exert a core force shielding effect which increases the local dislocation density and then increases the local stress field at the 


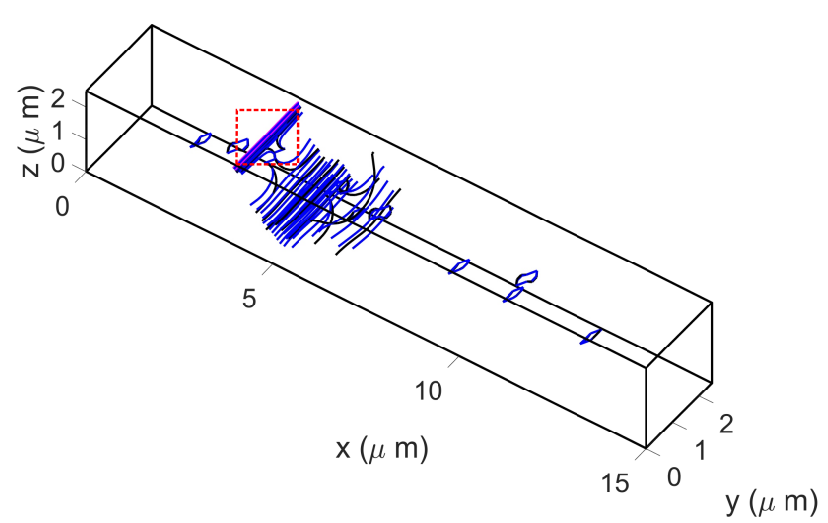

(a)

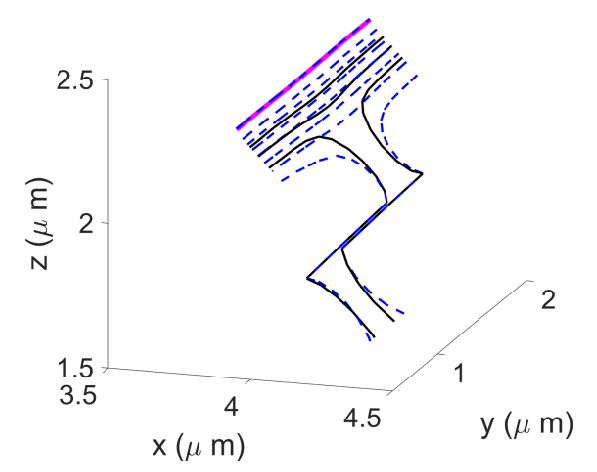

(b)

Fig. 12 (a) Dislocation structures with an obstacle (magenta) with hydrogen (blue dashed) and without hydrogen (black solid). (b) Zoom-in on the (red dashed-box) region near the obstacle in (a).

head of the pile-up, promoting failure at the obstacle. This tentatively supports the hydrogen-enhanced plasticity-mediated decohesion mechanism [5] however hydrogen enhanced dislocation activity is only feasible mechanism for hydrogen induced inter-granular failure observed in experiments [46, 47]. More likely is the synergistic action of a number of mechanisms, such as hydrogen enhanced dislocation activity and hydrogen enhanced decohesion [48].

These results show hydrogen core force shielding promotes dislocation activity and tends to reduce the flow stress in fcc materials, this has been observed in 316L stainless steel [49] and a nickel based 718 alloy $[50,51]$. However details relating to microstructure and loading condition will need to be taken into account to simulate experiments. For instance, Harris et al. [52] observed hydrogen increased the flow stress in polycrystalline pure nickel, Girardin and Delafosse [53] and Ghermaoui et al. [54] observed hydrogen mitigated hardening in single crystal nickel oriented for single and multiple slip, and Castelluccio et al. [55] concluded that the actual influence of hydrogen on the flow stress in nickel is dependent on the loading condition. Although the simulation of real experiments is beyond the current scope, it revealed that hydrogen core force shielding rather than elastic shielding influences plasticity at a realistic bulk concentration.

\section{Summary}

Hydrogen informed discrete dislocation plasticity simulations of the precursor to hydrogen induced failure was carried out for an fcc material. Both hydrogen elastic shielding [30] and the effect of hydrogen on the dislocation core energy [34] were implemented and evaluated. The latter was implemented in the form of a hydrogen core force in the simulations, which counteracts the original dislocation core force. It is therefore referred to as the hydrogen core force shielding mechanism.

Simulations were performed on a FR source in an infinite medium. Hydrogen is shown to promote the activation of screw FR sources and accelerate the expansion of dislocations loops generated from both edge and screw sources. This promotes the generation of dislocations and decreases the average spacing in a edge dislocation pile-up. In this process, hydrogen core force shielding is dominant, whereas the contribution of hydrogen elastic shielding is negligible, at realistic hydrogen concentrations.

In the microcantilever simulations, hydrogen is observed to increase dislocation density and soften global flow behavior, which agrees with the HELP theory. In addition, very clean dislocation pile-up structures are obtained close to the neutral axis, and hydrogen is shown to increase the pile-up density. When an obstacle is included near a free surface to prevent segments exiting, a dislocation pile-up is formed. This pile-up generates a stress field with the same sign as the corrective field (tensile) above the slip plane. By increasing the density of dislocations in the pile-up, hydrogen is observed to elevate the total tensile stress above the slip plane and therefore 


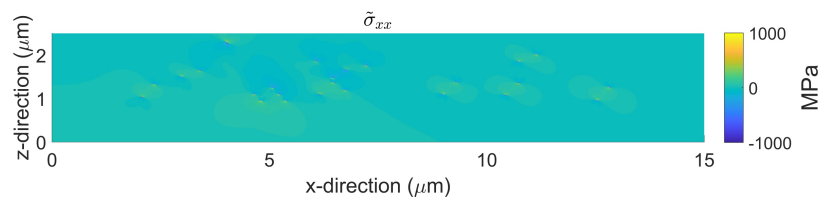

(a)

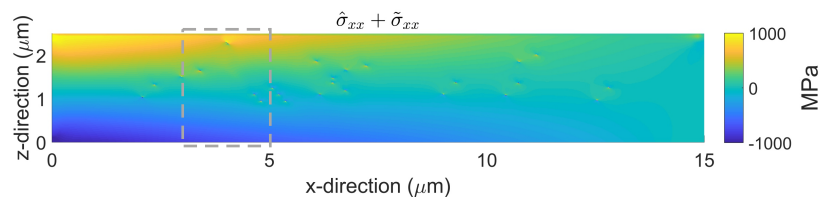

(c)

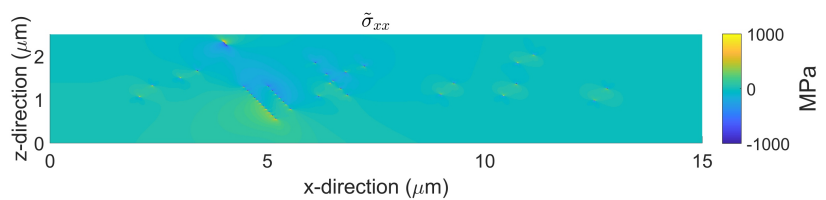

(b)

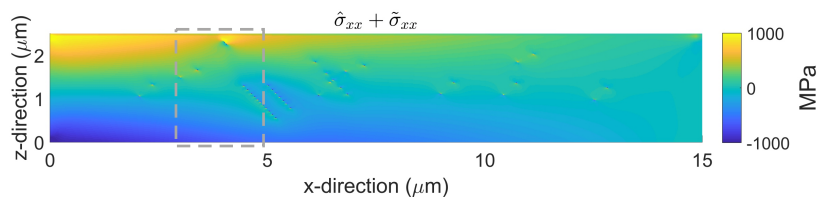

(d)

Fig. 13 Axial stress contours at $U=-0.19 \mu \mathrm{m}$ due to dislocations $\tilde{\sigma}_{x x}$ (a) and (b), and the superposition of the the dislocation and the corrective field $\hat{\sigma}_{x x}+\tilde{\sigma}_{x x}$ (b) and (c). (a) and (c) are obtained without hydrogen, and (b) and (d) are obtained with hydrogen. The stress generated by the obstacle has been excluded. The boxed regions in (c) and (d) is shown in Fig. 14.

facilitate stress-controlled failure.

This work indicates a hydrogen promoted stresscontrolled failure mechanism that could be generalized to a variety of cases, e.g. interfacial separation at an inclusion and grain boundary decohesion. This could lead to hydrogen-enhanced plasticity mediated intergranular failure [5]. However there exists a gap between the observed hydrogen enhanced plasticity and hydrogen induced failure To simulate hydrogenenhanced plasticity-mediated failure, a damage model needs to be incorporated. At present, the hydrogen core force $\left(\overline{\boldsymbol{F}}_{c}^{k}\right.$ in Equation 1) arises from solute-solute interactions in the near-core region. Solute-solute interactions can also contribute to the far-field hydrogen elastic force $\left(\overline{\boldsymbol{F}}^{k}\right)$, which could magnify the observed effects of hydrogen. The interactions outside the core regime will be implemented in the future.

\section{Acknowledgements}

This work was supported by the Engineering and Physical Sciences Research Council through Programme grant EP/L014742/1 and Fellowship grant EP/N007239/1. We also thank Prof. Jaafar El-Awady for a useful discussion at the Hydrogen in Metals Workshop in Oxford.

\section{References}

[1] I. Robertson, P. Sofronis, A. Nagao, M. L. Martin, S. Wang, D. W. Gross, K. E. Nygren, Hydrogen embrit- tlement understood, Metallurgical and Materials Transactions B 46 (2015) 1085-1103.

[2] R. A. Oriani, A mechanistic theory of hydrogen embrittlement of steels, Berichte der Bunsengesellschaft fr physikalische Chemie 76 (1972) 848-857.

[3] H. K. Birnbaum, P. Sofronis, Hydrogen-enhanced localized plasticitya mechanism for hydrogen-related fracture, Materials Science and Engineering: A 176 (1994) 191202.

[4] M. B. Djukic, G. M. Bakic, V. S. Zeravcic, B. Rajicic, A. Sedmak, R. Mitrovic, Z. Miskovic, Towards a unified and practical industrial model for prediction of hydrogen embrittlement and damage in steels, Procedia Structural Integrity 2 (2016) 604-611.

[5] A. Nagao, C. D. Smith, M. Dadfarnia, P. Sofronis, I. M. Robertson, The role of hydrogen in hydrogen embrittlement fracture of lath martensitic steel, Acta Materialia 60 (2012) 5182-5189.

[6] Y.-S. Chen, D. Haley, S. S. A. Gerstl, A. J. London, F. Sweeney, R. A. Wepf, W. M. Rainforth, P. A. J. Bagot, M. P. Moody, Direct observation of individual hydrogen atoms at trapping sites in a ferritic steel, Science 355 (2017) 1196-1199.

[7] T. Hajilou, M. S. B. Hope, A. H. Zavieh, N. Kheradmand, R. Johnsen, A. Barnoush, In situ small-scale hydrogen embrittlement testing made easy: An electrolyte for preserving surface integrity at nano-scale during hydrogen charging, International Journal of Hydrogen Energy 43 (2018) 12516-12529.

[8] X. Lu, Y. Ma, M. Zamanzade, Y. Deng, D. Wang, W. Bleck, W. W. Song, A. Barnoush, Insight into hydrogen effect on a duplex medium-mn steel revealed by in-situ nanoindentation test, International Journal of Hydrogen Energy 44 (2019) 20545-20551.

[9] M. Wang, E. Akiyama, K. Tsuzaki, Fracture criterion for hydrogen embrittlement of high strength steel, Materials Science and Technology 22 (2006) 167-172. 


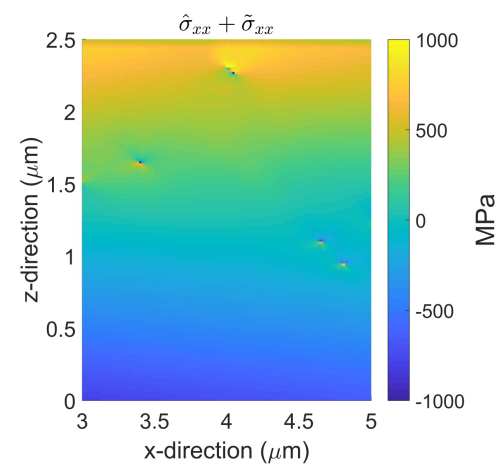

(a)

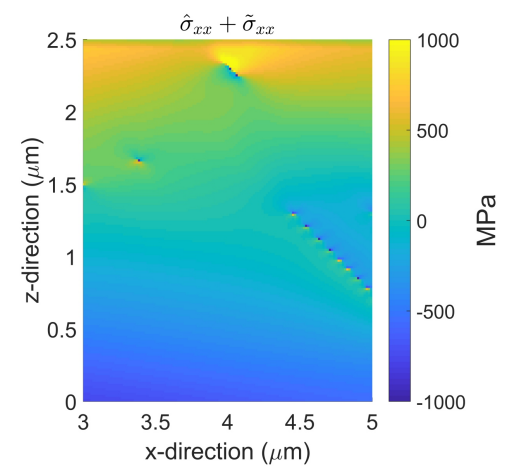

(b)

Fig. 14 The total stress field near the obstacle (the boxed regions in the in Fig. 13). (a) without hydrogen and (b) with hydrogen. The stress generated by the obstacle has been excluded.

[10] D. E. Jiang, E. A. Carter, First principles assessment of ideal fracture energies of materials with mobile impurities: implications for hydrogen embrittlement of metals, Corrosion Reviews 52 (2004) 4801-4807.

[11] S. Wang, N. Hashimoto, S. Ohnuki, Hydrogen-induced change in core structures of 110[111] edge and 110[111] screw dislocations in iron, Scientific Reports 3 (2013) 2760.

[12] S. Wang, K. Takahashi, N. Hashimoto, S. Isobe, S. Ohnuki, Strain field of interstitial hydrogen atom in body-centered cubic iron and its effect on hydrogendislocation interaction, Scripta Materialia 68 (2013) 249-252.

[13] Y. Zhao, G. Lu, Qm/mm study of dislocationhydrogen/helium interactions in $\alpha$-fe, Modelling and Simulation in Materials Science and Engineering 19 (2011) 065004.

[14] J. Song, W. A. Curtin, Mechanisms of hydrogenenhanced localized plasticity: An atomistic study using $\alpha$-fe as a model system, Acta Materialia 68 (2014) 6169.

[15] J. Song, W. Curtin, Atomic mechanism and prediction of hydrogen embrittlement in iron, Nature materials 12 (2013) 145.

[16] P. Sofronis, Y. Liang, N. Aravas, Hydrogen induced shear localization of the plastic flow in metals and alloys, European Journal of Mechanics - A/Solids 20 (2001) 857-872.

[17] D. C. Ahn, P. Sofronis, R. H. Dodds Jr, On hydrogeninduced plastic flow localization during void growth and coalescence, International Journal of Hydrogen Energy 32 (2007) 3734-3742.

[18] Y. Liang, D. C. Ahn, P. Sofronis, R. H. Dodds Jr, D. Bammann, Effect of hydrogen trapping on void growth and coalescence in metals and alloys, Mechanics of Materials 40 (2008) 115-132.

[19] O. Barrera, E. Tarleton, H. W. Tang, A. C. F. Cocks, Modelling the coupling between hydrogen diffusion and the mechanical behaviour of metals, Computational Materials Science 122 (2016) 219-228.

[20] H. Yu, J. S. Olsen, J. He, Z. Zhang, Hydrogen-microvoid interactions at continuum scale, International Journal of Hydrogen Energy (2018).

[21] S. Aubry, S. P. Fitzgerald, S. L. Dudarev, W. Cai, Equilibrium shape of dislocation shear loops in anisotropic $\alpha$-fe, Modelling and Simulation in Materials Science and Engineering 19 (2011) 065006.

[22] L. Capolungo, Dislocation junction formation and strength in magnesium, Acta Materialia 59 (2011) 29092917.

[23] S. P. Fitzgerald, S. Aubry, S. L. Dudarev, W. Cai, Dislocation dynamics simulation of frank-read sources in anisotropic $\alpha$-fe, Modelling and Simulation in Materials Science and Engineering 20 (2012) 045022.

[24] C. Motz, D. Weygand, J. Senger, P. Gumbsch, Microbending tests: A comparison between three-dimensional discrete dislocation dynamics simulations and experiments, Acta Materialia 56 (2008) 1942-1955.

[25] C. Motz, D. Weygand, J. Senger, P. Gumbsch, Initial dislocation structures in 3-d discrete dislocation dynamics and their influence on microscale plasticity, Acta Materialia 57 (2009) 1744-1754.

[26] I. Ryu, W. D. Nix, W. Cai, Plasticity of bec micropillars controlled by competition between dislocation multiplication and depletion, Acta Materialia 61 (2013) 3233-3241.

[27] Y. Cui, G. Po, N. Ghoniem, Temperature insensitivity of the flow stress in body-centered cubic micropillar crystals, Acta Materialia 108 (2016) 128-137.

[28] Y. Cui, G. Po, N. Ghoniem, Does irradiation enhance or inhibit strain bursts at the submicron scale?, Acta Materialia 132 (2017) 285-297.

[29] Q. Chen, X. Y. Liu, S. B. Biner, Solute and dislocation junction interactions, Acta Materialia 56 (2008) 29372947.

[30] Y. Gu, J. A. El-Awady, Quantifying the effect of hydrogen on dislocation dynamics: A three-dimensional discrete dislocation dynamics framework, Journal of the Mechanics and Physics of Solids (2018).

[31] W. Cai, R. B. Sills, D. M. Barnett, W. D. Nix, Modeling a distribution of point defects as misfitting inclusions in stressed solids, Journal of the Mechanics and Physics of Solids 66 (2014) 154-171. 
[32] H. Yu, A. Cocks, E. Tarleton, Discrete dislocation plasticity helps understand hydrogen effects in bcc materials, Journal of the Mechanics and Physics of Solids 123 (2019) 41-60.

[33] H. Yu, A. C. Cocks, E. Tarleton, The influence of hydrogen on lomer junctions, Scripta Materialia 166 (2019) $173-177$.

[34] R. B. Sills, W. Cai, Free energy change of a dislocation due to a cottrell atmosphere, Philosophical Magazine 98 (2018) 1491-1510.

[35] A. Arsenlis, W. Cai, M. Tang, M. Rhee, T. Oppelstrup, G. Hommes, T. G. Pierce, V. V. Bulatov, Enabling strain hardening simulations with dislocation dynamics, Modelling and Simulation in Materials Science and Engineering 15 (2007) 553.

[36] E. V. d. Giessen, A. Needleman, Discrete dislocation plasticity: a simple planar model, Modelling and Simulation in Materials Science and Engineering 3 (1995) 689.

[37] Y. Hu, B. Szajewski, D. Rodney, W. Curtin, Atomistic dislocation core energies and calibration of non-singular discrete dislocation dynamics, Modelling and Simulation in Materials Science and Engineering 28 (2019) 015005.

[38] V. Bulatov, W. Cai, Computer simulations of dislocations, volume 3, Oxford University Press on Demand, 2006.

[39] D. Delafosse, 9 - Hydrogen effects on the plasticity of face centred cubic (fcc) crystals, volume 1, Woodhead Publishing, pp. 247-285.

[40] J. Chateau, D. Delafosse, T. Magnin, Numerical simulations of hydrogen-dislocation interactions in fcc stainless steels.: part ii: hydrogen effects on crack tip plasticity at a stress corrosion crack, Acta Materialia 50 (2002) 15231538.

[41] W. Cai, Test case: Frank-read source, http: //micro.stanford.edu/wiki/Test_Case: _Frank-Read_Source, Web Page. Accessed: 2019-0617.

[42] P. Anderson, J. Hirth, J. Lothe, Theory of Dislocations, Cambridge University Press, 2017.

[43] E. Tarleton, D. S. Balint, J. Gong, A. J. Wilkinson, A discrete dislocation plasticity study of the micro-cantilever size effect, Acta Materialia 88 (2015) 271-282.

[44] I. M. Robertson, The effect of hydrogen on dislocation dynamics, Engineering Fracture Mechanics 68 (2001) 671692.

[45] D. Wang, X. Lu, Y. Deng, X. Guo, A. Barnoush, Effect of hydrogen on nanomechanical properties in fe-22mn-0.6 c twip steel revealed by in-situ electrochemical nanoindentation, Acta Materialia 166 (2019) 618-629.

[46] M. L. Martin, B. P. Somerday, R. O. Ritchie, P. Sofronis, I. M. Robertson, Hydrogen-induced intergranular failure in nickel revisited, Acta Materialia 60 (2012) 2739-2745.

[47] S. Wang, M. L. Martin, P. Sofronis, S. Ohnuki, N. Hashimoto, I. M. Robertson, Hydrogen-induced intergranular failure of iron, Acta Materialia 69 (2014) 275282.

[48] M. B. Djukic, G. M. Bakic, V. Sijacki Zeravcic, A. Sed- mak, B. Rajicic, The synergistic action and interplay of hydrogen embrittlement mechanisms in steels and iron: Localized plasticity and decohesion, Engineering Fracture Mechanics 216 (2019) 106528.

[49] Y. Kim, D. Shin, Y. Kim, D. W. Kim, S. Kim, W. Nam, Y.-S. Kim, K. Mthis, H. Choe, Hydrogen softening in the thin plate of microcrystalline 3161 stainless steel, steel research international 84 (2013) 812-817.

[50] G. Stenerud, R. Johnsen, J. S. Olsen, J. He, A. Barnoush, Effect of hydrogen on dislocation nucleation in alloy 718, International Journal of Hydrogen Energy 42 (2017) 15933-15942.

[51] Z. Tarzimoghadam, D. Ponge, J. Klwer, D. Raabe, Hydrogen-assisted failure in ni-based superalloy 718 studied under in situ hydrogen charging: The role of localized deformation in crack propagation, Acta Materialia 128 (2017) 365-374.

[52] Z. D. Harris, S. K. Lawrence, D. L. Medlin, G. Guetard, J. T. Burns, B. P. Somerday, Elucidating the contribution of mobile hydrogen-deformation interactions to hydrogen-induced intergranular cracking in polycrystalline nickel, Acta Materialia 158 (2018) 180-192.

[53] G. Girardin, D. Delafosse, Solute-dislocation interactions: modelling and experiments in hydrogenated nickel and nickel base alloys, Materials Science and Engineering: A 387-389 (2004) 51-54.

[54] I. M. A. Ghermaoui, A. Oudriss, A. Metsue, R. Milet, K. Madani, X. Feaugas, Multiscale analysis of hydrogeninduced softening in f.c.c. nickel single crystals oriented for multiple-slips: elastic screening effect, Scientific Reports 9 (2019) 13042.

[55] G. M. Castelluccio, C. B. Geller, D. L. McDowell, A rationale for modeling hydrogen effects on plastic deformation across scales in fcc metals, International Journal of Plasticity 111 (2018) 72-84. 